\title{
Pneumatic Classification of Granular Materials in Rhomb-Shaped Apparatus
}

\author{
Mykola Yukhymenko ${ }^{1}$, Ruslan Ostroha ${ }^{1, *}$ and Artem Evtuhov ${ }^{1}$ \\ Sumy State University, 2 Rymskogo-Korsakova St., 40007 Sumy, Ukraine
}

Abstract: The article deals with studying of the gas-dispersed systems classification process in gravitation pneumoclassifiers of prismatic shape. The research is aimed at developing a pneumatic classification method for granular materials using a rhomb-shaped apparatus and ensuring the reliability of the operating process based on the influence of the flow on the granular material concentrations. The related mathematical model is developed for estimating the non-stationary concentration of fine particles in a gas-dispersed flow with respect to time and height of the working space of the apparatus. It is shown that the rhomb-shaped pneumatic classifier provides effective separation of granular material, reaching up to $95 \%$ of the target fraction.

Key words: pneumoclassifier; granular material; weighted layer, fine particles entrainment; fine particle concentration; mathematical modeling; Laplace transform

\section{Introduction}

Removal of highly dispersed particles from a mass of various polydispersed materials (dedusting) is a widespread technological operation that enables to eliminate dust emission and dust entrainment during the subsequent processing of granular materials. After the removal of highly dispersed particles, energy consumption for grinding the material is reduced, the gas permeability and sintering capacity of dust-free granular masses increases; their shape, flowability, and intensity of mechanical classification are much better. If granular materials of homogeneous chemical composition are separated with a gas flow into two or more fractions, this process is called pneumatic classification. It is used both as an independent technological operation (sorting) and as a preparation of granular material for subsequent processing.

Known designs of pneumatic classifiers are effectively used for separating the products when the content of fine fractions in the initial material is maximum 15$20 \%$. When separating binary free-flowing mixtures that contain equal amount of fine and coarse fractions, typical pneumatic classifiers provide purity of the obtained fractions up to a maximum of $70-75 \%$. However, there are technologies that require the production of pure separation products with the purity of at least $95 \%$.

Therefore, a necessary task is to develop new methods and designs of pneumatic classifiers by rational use of the working volume and implementation of more effective methods of influencing the granular material flow. This technique will contribute to additional sieving of the material and, thus, significantly improve separation of the polydispersed granular mixture into fine and coarse fractions.

\section{Problem Statement}

In work [1] an overview of modern air classification devices is given; their operation principles, features and parameters, as cut size, cleanness and recovery are presented. It is proven, that in practice, various gravitational and centrifugal classifiers are employed,

*Corresponding author: Ruslan Ostroha, E-mail address: r.ostroga@pohnp.sumdu.edu.ua 
including those with vertical and horizontal air streams, cascade classifiers, fluidized bed separators, inertial, vortex, rotor classifiers, etc.

Centrifugal pneumatic classifiers are structurally complex and contain a block of separation elements [2] or they have rotating rotor with blades [3], which requires balancing the shaft.

Pneumatic classifiers based on the action of centrifugal forces are mainly used for separation of fine materials with a particle size less than 0.04-0.05 $\mathrm{mm}[4,5]$. Their main disadvantage is the content of a significant amount (about 20-25\%) of fine particles and the presence of a coarse fraction in the final product.

The gravitational pneumatic classification method provides higher operational reliability [6].

In works [7, 8], the process of pneumatic separation of polydispersed granular mixture consisting of three fractions $(4.75-8 \mathrm{~mm}, 8-12.7$ $\mathrm{mm}, 12.7-19.1 \mathrm{~mm}$ ) was investigated. Air blowing of the granular layer makes it possible to separate the product into different fractions. However, it is indicated that after separation the coarse product is contaminated with fine particles, and the coarse fraction gets into the fine product. Final products require additional mechanical sieving after pneumatic classification.

The simplest pneumatic classifiers with vertical channels are not widely used since they do not provide the required separation efficiency [9]. In such devices, a raw material is loaded into the working volume, causing an increase in gasdispersed flow density. Consequently, the mixture has poor contact with the gas flow. Therefore, the coarse fraction entrains the fine fraction, which decreases the efficiency of fractional separation.

Further development of such devices was presented by zigzag pneumatic classifiers $[10,11]$, wherein centrifugal forces arise due to the gas flow turns, causing particle circulation. This effect reduces flow density and, consequently, increases the efficiency of the classification process. However, this type of pneumatic classifier was modernized, and the new design has a vertical channel with a rectangular cross-section, in which blind shelves are installed at two angles to the gas flow $[12,13]$. However, blind shelves significantly increase the hydraulic resistance of the device and turbulize the flow, which increases entrainment of a coarse fraction into the cyclone. The device with inclined perforated shelves allows implementing an active aerodynamic mode of suspending the granular layer. Consequently, entrainment of the coarse fraction and energy costs of the pneumatic classification process are reduced.

The apparatus of this design enables effectively removing the fine fraction of less than $1 \mathrm{~mm}$ from the polydispersed mixture of granular mineral fertilizers [14]. Pneumatic classifiers with a fluidized bed of the granular layer and perforated grate [15, 16] are mainly intended for separation of the bulk product in order to separate particles less than $0.5 \mathrm{~mm}$ from the layer. This allows extending the separation possibility to significantly increase the gas flow rate, which leads to increased energy costs.

Thus, a rhomb-shaped pneumatic classifier is a relatively new device, which is applied in the technology of producing organic mineral fertilizers $[17,18]$. Due to the absence of internal contact elements, the rhomb-shaped pneumatic classifiers have a minimized hydraulic resistance. Moreover, a relatively high degree of product separation in such an apparatus is achieved by cyclic loading of raw material into the apparatus, thereby creating a rotating vortex layer; accordingly, prolongation of the contact of particles with the gas flow is efficiently provided.

\section{Experimental Materials and Methods}

Experimental studies of granular material separation into fractions were performed in the developed experimental laboratory stand (Fig. 1).

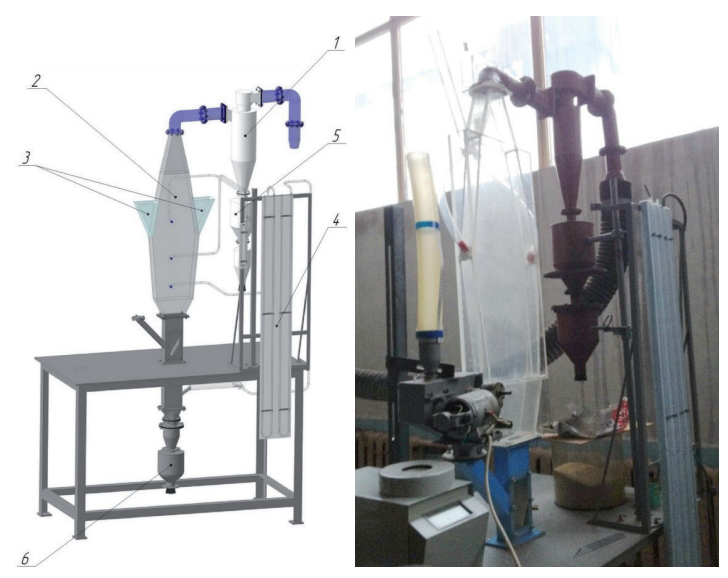

Figure 1: Scheme (a) and photograph (b) of the experimental stand for pneumatic classification: 1 - cyclone; 2 - pneumatic classifier; 3 - loading hoppers for raw materials; 4 manometers; 5 - fine fraction tank; 6 - coarse fraction tank 
The laboratory stand (Fig. 1) includes a pneumatic classifier 2, which is made of organic glass, that improves visualization of the process studied. Separation takes place directly in the housing of the pneumatic classifier, which has 2 working sections, the lower section is the separation zone, in which the suspended layer is rotated and the material is sieved, as well as coarse and fine fractions removal takes place; the upper section is the zone wherein the fine fraction is accelerated and removed from the device. The fine fraction removed from the apparatus passes through the cyclone 1 and goes into the fine fraction tank 5 , and the coarse fraction falls down and gets into the coarse fraction tank 6 . Loading of raw material is fulfilled through loading hoppers 3.

Quartz sand was used as a model material. Using a set of sieves ( $0.63 \mathrm{~mm} ; 0.4 \mathrm{~mm} ; 0.315 \mathrm{~mm} ; 0.2 \mathrm{~mm}$; $0.16 \mathrm{~mm}$ ) one prepared binary mixture containing of fractions $0.16-0.2 \mathrm{~mm}(50.91 \%)$ and fractions 0.40-0.63 mm (49.09\%). Additionally, stand efficiency was tested on the following materials: urea (fractions 0.2-2.0 mm - 48.7\%; 2.0-4.0 mm $51.3 \%$ ) and seeds of agricultural crops (buckwheat, millet, carrot, and radish).

Quartz sand was dried in a muffle furnace and using a set of sieves $+0.63 \mathrm{~mm} ;+0.40 \mathrm{~mm} ;+0.315$ $\mathrm{mm} ;+0.20 \mathrm{~mm} ;+0.16 \mathrm{~mm}$, a charge of sand was obtained. Then the charge was weighed on an electronic scale of Momert-6000 with an accuracy of $0.1 \mathrm{~g}$ and loaded into the hopper for raw material feeding. Further, the gas blower was switched on, and the air flow was controlled by means of a clamp pressing the air flow.

Pressure in the apparatus body was fixed with the help of U-shaped liquid pressure gauges. Air velocity in the device was measured using Pitot-Prandtl's tubes inserted at different cross sections of the body. The tubes were connected to a micromanometer with a sloping tube MMN240(5)-0.1, which measured the excess pressure at the cross sections of the apparatus body; and the pressure value, with the help of tare graphs, was translated into velocity. Certain charges of the material were periodically loaded into the device. To start a process, a triple amount of material was fed into the device to create a rotating layer in the body. Then the loading hopper cover was opened every 60 seconds and the next amount of material in the volume of 50-60 grams was fed into the body in 15 seconds. It created the specific load rate within the limits of $18-65 \mathrm{~kg} / \mathrm{m} 2 \cdot \mathrm{s}$. The layer rotating in the body was again filled with material and the process was stabilized.

\section{Results}

To organize the process of pneumatic classification in a hollow apparatus of variable crosssection it is necessary to provide active contact of the particle with the air flow. In our case, we have a suspended layer of rotating material, which is formed by feeding a first triple dose of material into the apparatus. Separation efficiency depends on the rotation velocity and mass of the suspended layer. In general, the rotating suspended layer is shown in Fig. 2, a.
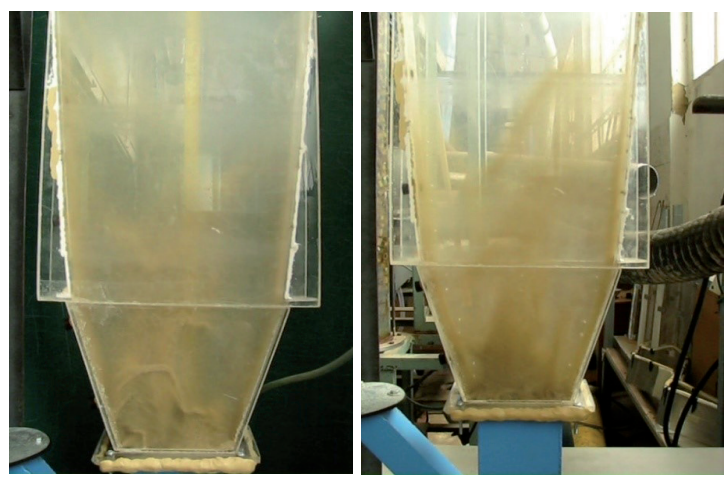

Figure 2: General view of the rotating suspended layer in the apparatus working volume

Based on the process peculiarities, it can be seen that there is a dynamic displacement of the suspended layer centre along certain trajectories, which is shown in Fig. 2, b. Due to the suspended layer rotation, the particles of material are sent to the desired area of the apparatus, either to the separation zone, or to the zone of acceleration and removal of fine particles from the apparatus. At the time of loading the participles into the apparatus, fine fractions are immediately blown out by the air flow, and coarse fractions fall into the suspended layer.

At the moment of the suspended layer rotation, coarse particles fall out of the flow and are removed from the bottom of the apparatus, and fine particles, gaining rotation velocity of the suspended layer and speeded up by air flow, get into the upper zone wherein they are accelerated and removed from the apparatus with the air flow. 
The main criterion for evaluating the operation of the apparatus for pneumatic classification is the separation efficiency, which is defined as the loss of a coarse fraction, i.e. the removal of this fraction by the gas flow from the apparatus.

To analyse the influence of hydrodynamic parameters of the gas flow on the separation efficiency, the dependence of the coarse fraction losses of granular material on the gas flow consumption rate (Fig. 3) and the gas flow velocity (Fig. 4) is made.

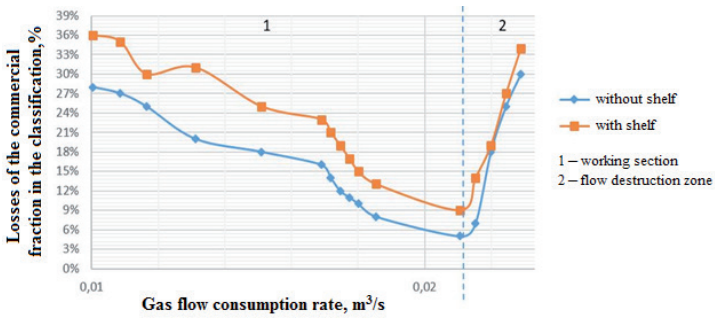

Figure 3: Dependence of the coarse fraction losses of granular material on the gas flow consumption rate

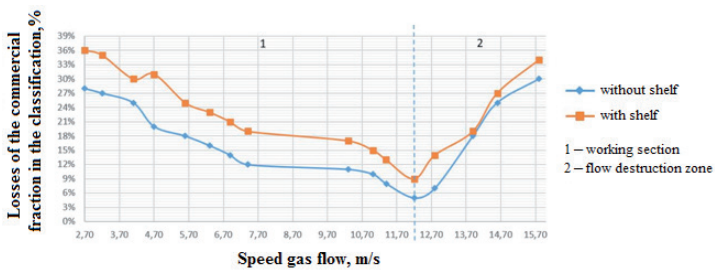

Figure 4: Dependence of the commercial fraction losses of the granular material on the speed gas flow

\section{Discussion}

As it can be seen from the dependences (Fig. 3, 4), we figuratively obtain 2 sections in which we can evaluate the influence of the gas flow consumption rate. In the first section (working zone) there is a dynamic of reducing the coarse fraction losses and less contamination of a coarse fraction with the fine fraction with increasing the gas flow consumption rate, which has a positive effect on the efficiency of classification. However, this trend continues until the flow consumption rate exceeds $0.024748 \mathrm{~m}^{3} / \mathrm{s}$ and the flow velocity is $12 \mathrm{~m} / \mathrm{s}$. At these parameters we will obtain contamination of a coarse fraction with the fine fraction within $5 \%$. With a further increase in the gas flow consumption rate (more than $0.024748 \mathrm{~m}^{3} / \mathrm{s}$ and $12 \mathrm{~m} / \mathrm{s}$, see the second zone of the diagram - flow destruction zone), there is a gradual destruction of the rotating layer of material and removal of particles of coarse fraction with the gas flow from the apparatus.

Select the elementary volume in the suspended layer of material in the apparatus (Fig. 5).

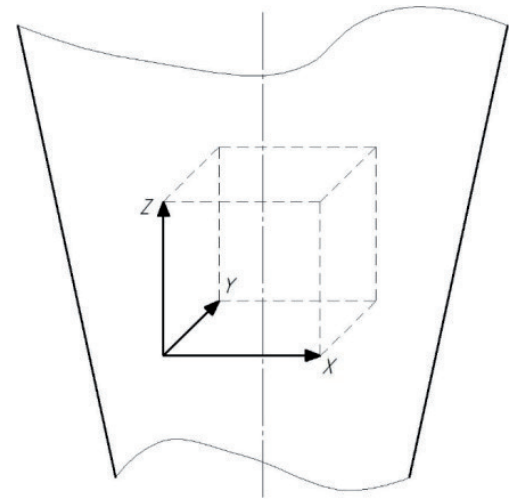

Figure 5: Schematic drawing of elementary volume in the apparatus

Let's consider the change in the concentration of fine fraction particles (CM) in the taken volume in time and space. The equation describing this change is expressed as:

$G_{l} \frac{\partial C_{M}(\tau)}{\partial \tau}=G_{l}\left(U_{T} \operatorname{grad}_{M}\right)+G_{l} K_{y}\left(C_{M}^{*}-C_{M}(\tau)\right)=$
$=\left(U_{T_{X}} \frac{\partial C_{M}}{\partial X}+U_{T_{Y}} \frac{\partial C_{M}}{\partial Y}+U_{T_{Z}} \frac{\partial C_{M}}{\partial Z}\right)+G_{l} K_{y}\left(C_{M}^{*}-C_{M}(\tau)\right)$,

where $G_{l}$ - mass of particles in the suspended layer, $\mathrm{kg} ; U_{T x}, U_{T y}, U_{T z}$ - solid particles velocity in accordance with the axes $X, Y, Z, m / s ; C M(\tau)$ concentration of fine particles in the suspended layer, $\mathrm{kg} / \mathrm{kg} ; C^{*}{ }_{M}$ - limiting concentration of fine particles in the suspended state, $\mathrm{kg} / \mathrm{kg}$; $\tau$ - time of the pneumatic classification process, $s ; K_{y}$ - constant of entrainment rate, $1 / \mathrm{s}$.

Due to the fact that the width of the pneumatic classifier is much less than the length of the cross section, we assume that there are no changes in the concentration of fine particles along the $Y$ axis, ie in Eq. (1) we take

$U_{T_{Y}} \frac{\partial C_{M}}{\partial Y}=0$

Also, since the velocity of solid particles in the horizontal projection $\mathrm{X}$ in the direction from the axis 
of the apparatus to the walls is significantly reduced due to the braking effect and for the same reason the gas flow in the horizontal direction will not significantly affect particle stratification, then in Eq. (1) we also obtain:

$$
U_{T_{X}} \frac{\partial C_{M}}{\partial X}=0
$$

After these assumptions, Eq. (1) will be written as:

$$
G_{l} \frac{\partial C_{M}(\tau)}{\partial \tau}=G_{l} U_{T_{Z}} \frac{\partial C_{M}}{\partial Z}+G_{l} K_{y}\left(C_{M}^{*}-C_{M}(\tau)\right)
$$

We present Eq. (4) in the form:

$$
\frac{\partial C_{M}(Z, \tau)}{\partial \tau}+U_{T_{Z}} \frac{\partial C_{M}(Z, \tau)}{\partial Z}-K_{y}\left[C_{M}^{*}-C_{M}(Z, \tau)\right]=0
$$

The solution of Eq. (5) by the Laplace transform method obtained in [19] is represented as:

$$
\begin{aligned}
C_{M}(Z, \tau) & =C_{M}^{*}+\left(C_{0}-C_{M}^{*}\right) \\
& \left\{e^{\frac{-k Z}{U_{T}}} \eta(\tau)\left(\tau-\frac{Z}{U_{T}}\right)+e^{-k \tau}\left[1-\eta(\tau)\left(\tau-\frac{Z}{U_{T}}\right)\right]\right\}
\end{aligned}
$$

where $C_{0}$ is the initial concentration of fine particles, $\mathrm{kg} / \mathrm{kg} ; \eta(\tau)$ is the Heaviside step function, for $\tau \geq 0$ : $\eta(\tau)=1$.

Using Eq. (6), we make the graph of changes of fine particles concentration in the suspended layer along the height of the apparatus Z over time $\mathrm{\tau}$ (Fig. 6).

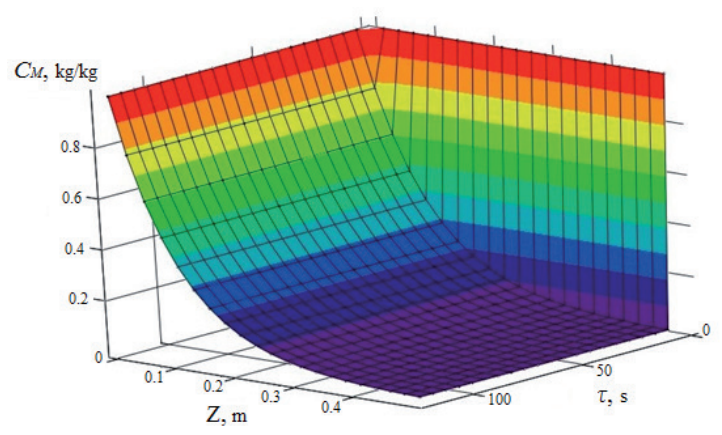

Figure 6: Dependence of the change in the concentration of fine particles in the suspended layer on the height of the apparatus Z over time $\tau$

The obtained results prove the fact that the concentration of fine particles is reduced by the apparatus height in time. This fact allows ensuring the separation efficiency.

\section{Conclusions}

The proposed physical model of granular mixture separation in rhomb-shaped pneumatic classifiers allows explaining the hydrodynamic features of separation into fractions. The related mathematical model of the classification processes of granular mixtures was developed to obtain the generalized equation for determining the concentration of a fine fraction in a suspended layer. A cyclic method for carrying out the pneumatic separation process is proposed. The initial cycle consists of loading a suspended layer with the outlet mixture for 15-20 s. The next cycle involves gradual unloading of the material accumulated in the layer.

The experimental research on the separation efficiency for granular material also showed the presence of two different sections. In the first section, loss of the coarse fraction decreased, contamination of a coarse fraction by the fine particles was observed, and the flow rate consumption increased. In the second section, flow destruction occurred, and flow rate and loss of the coarse fraction increased. As a result, it was shown that the rhombshaped pneumatic classifier provides effective separation of granular material with the purity of the target fraction up to $95 \%$. Thus, contamination of a fine fraction by the coarse one does not exceed $5 \%$.

Further research should be aimed at checking the functionality and strength characteristics of the device using the finite element method [20, 21], as well as the reliability of the device design according to the methodology [22].

\section{Acknowledgments}

This research work has been supported by the Ministry of Science and Education of Ukraine under the project "Small-scale energysaving modules with the use of multifunctional devices with intensive hydrodynamics for the production, modification and encapsulation of granules" Project No. $0119 U 100834$.

\section{References}

1. Shapiro, M., Galperin, V. (2005). Air classification of solid particles: a review. Chemical Engineering and Processing: Process Intensification, 44, 2, 279-285.

2. Altun, O., Benzer, H. (2014). Selection and mathematical modelling of high efficiency air classifiers. Powder Technol- 
ogy, 264, 1-8.

3. Altun, O. (2016). Simulation aided flow sheet optimization of a cement grinding circuit by considering the quality measurements. Powder Technology, 301, 1242-1251.

4. Liu, R., Liu, J., Yu, Y. (2015). Effects of axial inclined guide vanes on a turbo air classifier. Powder Technology, 280, 1-9.

5. Ren, W., Liu, J., Yu, Y. (2016). Design of a rotor cage with nonradial arc blades for turbo air classifiers. Powder Technology, 292, 46-53.

6. Falconer, A. (2003). Gravity separation: old technique / new methods. Physical Separation in Science and Engineering, 12, 1, 31-48.

7. Ambrós, W. M., Sampaio, C. H., Cazacliu, B. G., Conceição, P. N., Glaydson S. R. (2019). Some observations on the influence of particle size and size distribution on stratification in pneumatic jigs. Powder Technology, 342, 594-606.

8. Ambrós, W. M. (2020). Jigging: a review of fundamentals and future directions. Minerals, 10, 998.

9. He, Y., Duan, C., Wang, H., Zhao, Y., Tao, D. (2011). Separation of metal laden waste using pulsating air dry material separator. Int. J. Environ. Sci. Technol., 8, 73-82.

10. Banjac, V., Pezo, L., Pezo, M., Vukmirovic, D., Colovic, D., Fistes, A., Covic, R. (2017). Optimization of the classification process in the zigzag air classifier for obtaining a high protein sunflower. Powder Technology, 28, 1069-1078.

11. Mann, H., Roloff, C., Hagemeier, T., Thevenin, D., Tomas, J. (2017) Model-based experimental data evaluation of separation efficiency of multistage coarse particle classification in a zigzag apparatus. Powder Technology, 313, 145-160.

12. Yukhymenko, M., Ostroha, R., Litvinenko, A., Bocko, J. (2017). Estimation of gas flow dustiness in the main pipelines of booster compressor stations. IOP Conf. Series: Materials Science and Engineering, 233, 012026.

13. Ponomarev, V. B., Shishkin, A. S. (2020). Methodology of designing an air cascade separator. IOP Conf. Series: Materials Science and Engineering, 966, 012043.

14. Yukhymenko, M., Artyukhov, A., Ostroha, R., Artyukhova, N., Krmela, J., Bocko, J. (2021) Multistage shelf devices with fluidized bed for heat-mass transfer processes: Experimental studies and practical implementation // Appl. Sci., 11, 3, 1159.

15. Liu, Z., Xie, Y., Wang, Y., Yu, J., Gao, S., Xu, G. (2012). Tandem fluidized bed elutriator - pneumatic classification of coal particles in a fluidized conveyer. Particuology, 10, 600-606.

16. Yang, G., Zheng, D., Zhou, J., Zhao, Y., Chen, Q. (2002). Air classification of moist raw coal in a vibrated fluidized bed. Miner. Eng., 15, 623-625.

17. Yukhymenko, M., Lytvynenko, A. (2014). Pneumatic classification of the granular materials in the "rhombic" apparatus. Journal of Manufacturing and Industrial Engineering, 1, 2,
$1-3$.

18. Lytvynenko, A., Yukhymenko, M., Pavlenko, I., Pitel, J., Mizakova, J., Lytvynenko, O., Ostroha, R., Bocko, J. (2019). Ensuring the reliability of pneumatic classification process for granular material in a rhomb-shaped apparatus. Appl. Sci., 9, 8, 1604.

19. Pavlenko I. V., Yukhymenko M. P., Lytvynenko A. V., Bocko J. (2019). Solving the nonstationary problem of the disperse phase concentration during the pneumoclassification process of mechanical mixtures. Journal of Engineering Sciences, 6, 1, F1-F5.

20. Trebuňa, F., Bocko, J., Pástor, M., Lengvarský, P., Prada, E. (2018). The finite element analysis of high precision positioning system. Strojnicky Casopis, 68, 4, 41-48.

21. Pástor, M., Bocko, J., Lengvarský, P., Prada, E. (2019). Numerical and experimental analysis of high precision positioning system. Experimental Stress Analysis - 57th International Scientific Conference, EAN 2019 - Conference Proceedings, 365-372.

22. Bocko, J., Delyová, I., Frankovský, P., Neumann, V. (2020). Lifetime assessment of the technological equipment for a robotic workplace. International Journal of Applied Mechanics, 12, 9, 2050097. 